\title{
Identifying undetected dementia in UK primary care patients: a retrospective case- control study comparing machine-learning and standard epidemiological approaches
}

Elizabeth Ford ${ }^{1 *}$ D, Philip Rooney ${ }^{2}$, Seb Oliver², Richard Hoile ${ }^{1}$, Peter Hurley², Sube Banerjee ${ }^{3}$, Harm van Marwijk ${ }^{1}$ and Jackie Cassell ${ }^{1}$

\begin{abstract}
Background: Identifying dementia early in time, using real world data, is a public health challenge. As only twothirds of people with dementia now ultimately receive a formal diagnosis in United Kingdom health systems and many receive it late in the disease process, there is ample room for improvement. The policy of the UK government and National Health Service (NHS) is to increase rates of timely dementia diagnosis. We used data from general practice (GP) patient records to create a machine-learning model to identify patients who have or who are developing dementia, but are currently undetected as having the condition by the GP.

Methods: We used electronic patient records from Clinical Practice Research Datalink (CPRD). Using a case-control design, we selected patients aged $>65 y$ with a diagnosis of dementia (cases) and matched them 1:1 by sex and age to patients with no evidence of dementia (controls). We developed a list of 70 clinical entities related to the onset of dementia and recorded in the 5 years before diagnosis. After creating binary features, we trialled machine learning classifiers to discriminate between cases and controls (logistic regression, naïve Bayes, support vector machines, random forest and neural networks). We examined the most important features contributing to discrimination.

Results: The final analysis included data on 93,120 patients, with a median age of 82.6 years; $64.8 \%$ were female. The naive Bayes model performed least well. The logistic regression, support vector machine, neural network and random forest performed very similarly with an AUROC of 0.74 . The top features retained in the logistic regression model were disorientation and wandering, behaviour change, schizophrenia, self-neglect, and difficulty managing.

Conclusions: Our model could aid GPs or health service planners with the early detection of dementia. Future work could improve the model by exploring the longitudinal nature of patient data and modelling decline in function over time.
\end{abstract}

Keywords: Dementia, General practice, Diagnosis, Prediction, Machine learning, Early detection, Primary care, Electronic health records

\footnotetext{
* Correspondence: e.m.ford@bsms.ac.uk; E.m.ford@bsms.ac.uk

1 Department of Primary Care and Public Health, Brighton and Sussex Medical

School, Watson Building, Village Way, Falmer, Brighton BN1 9PH, England

Full list of author information is available at the end of the article
}

(c) The Author(s). 2019 Open Access This article is distributed under the terms of the Creative Commons Attribution 4.0 International License (http://creativecommons.org/licenses/by/4.0/), which permits unrestricted use, distribution, and reproduction in any medium, provided you give appropriate credit to the original author(s) and the source, provide a link to the Creative Commons license, and indicate if changes were made. The Creative Commons Public Domain Dedication waiver (http://creativecommons.org/publicdomain/zero/1.0/) applies to the data made available in this article, unless otherwise stated. 


\section{Background}

Dementia encompasses a range of disorders characterised by progressive decline in memory, reasoning, communication and the ability to carry out daily activities $[1,2]$. The negative impact of this disorder on patients, their carers, family members and society is profound [3]. It can be hard to detect as patients may not present in healthcare clinics seeking a diagnosis. Around 850,000 people currently live with dementia in the United Kingdom (UK) [4]. Driven by population ageing this is projected to exceed 2,000,000 by 2051 [5]. With a prevalence of $7.1 \%$ in the over $65 \mathrm{~s}$ [5], better community care for people living with dementia is one of the great public health challenges of our era.

In the United Kingdom (UK), general practitioners (GPs) play a central role in the recognition and management of dementia in the community, and receive financial incentives for maintaining dementia registers and providing care. However, only around two-thirds of the expected numbers of patients with dementia are diagnosed [6] and recorded in GP dementia registers [7], and many of them only at an advanced stage. Data from Public Health England suggest that although diagnosis rates are increasing, they were still only $67.6 \%$ in March 2017, suggesting a third of patients are still not receiving a diagnosis [8].

Timely diagnosis for all people with dementia, who wish to have the diagnosis made, is a key objective of the UK National Dementia Strategy [2]. Timely diagnosis means that people with dementia can gain access to specialist assessment, treatment and support. Once diagnosed, patients can learn about the condition and plan for the future, which may help maximize quality of life and delay admission to care homes [9]. There is a need to improve detection and recording of dementia in UK general practice. Additional and innovative means of finding patients with dementia, based on actual local data, may improve diagnosis rates.

GPs record information about all interactions with their patients in electronic patient records (EPRs). These records consist of both structured (coded) and unstructured (free text) data entered into the patient record at the point of care. Some GP practices contribute the structured parts of their patient records in anonymised form to data warehouses such as the Clinical Practice Research Datalink (CPRD), which holds data on five million current patients [10]. Unlike traditional health research datasets, these routinely collected clinical data offer the opportunity to augment conventional health variables with multiple administrative and social variables (referrals, social care needs, etc), and with longitudinal patterns, such as changes in a patient's symptoms or medications over time, with high external validity to the real world. These records are frequently used by researchers for epidemiological studies or for monitoring post-marketing drug safety [11].

GP patient records could provide a valuable resource for improving the detection of dementia in general practice, and may provide a practical data source for creating diagnostic support algorithms for GPs. Retrospective studies have demonstrated significant differences in signs and symptoms found in the GP records of patients leading up to a dementia diagnosis compared to patients who do not go on to develop dementia [12, 13]. Cognitive symptoms, contact with social care professionals, unpredictable consulting patterns, increased attendance, level of carer involvement, and gait disturbance were all higher in patients who went on to be diagnosed with dementia within the next 5 years $[12,13]$.

While many studies have attempted to create clinical risk prediction models for dementia [14-18], only a few have tried to do this using only routinely collected general practice data [19-21], and none have been focused on early detection. One example of predicting future dementia risk from primary care data was presented by Walters et al. who created a clinical prediction model for dementia using only 14 clinical variables which performed poorly ( $\mathrm{C}$ index of $0.56)$ in patients over 80 years old, where risk is highest. It had good discrimination for 60-79 year olds ( $C$ index of 0.84 ), but various thresholds for high risk resulted in either a low sensitivity or a low positive predictive value $(0.11)$ [19]. A German primary care cohort of people 75 years of age and over, used even fewer variables (12) in a stepwise multivariate Cox proportional hazards model, achieving an AUC of 0.79 ; notably this used specific assessment procedures as predictors, which may add to clinic workload in routine primary care [20].

A further limitation of the current state of evidence is that is not clear which statistical methods work best when creating models with primary care data. Machine learning approaches have been trialled for predicting dementia, using predictors such as known clinical risk factors, dementia symptoms, and behaviours (such as missing appointments) [21]. One study found that a Naïve Bayes classifier gave the best result [21]. However, it incorporated a range of clinical information indicating that the GP had already picked up on dementia symptoms (e.g. codes for forgetfulness) and gave no information about the most important features in the model. To aid early detection, and to create models which could underpin dementia diagnostic support algorithms, it is important to develop models that can detect dementia before memory loss symptoms are noted by the GP.

In contrast to previous studies, our aim was to detect existing dementia before any evidence that the GP had done so, that is, before she or he had started recording memory loss symptoms or initiating the process of dementia diagnosis. We developed models, based on routine retrospective GP data, to best predict dementia caseness detected in usual care, using information in the five years before diagnosis (or matched date in controls). We aimed to improve on previous studies by (i) incorporating previously 
unused symptoms, medications, social and administrative variables ("clinical entities") as predictive features, and generating feature weights illustrating the most important predictors in the model; and (ii) comparing a range of machine learning techniques with a baseline approach of logistic regression.

\section{Methods}

\section{Data source}

This study used data from the UK Clinical Practice Research Datalink (CPRD) [22], established in 1987, which now contains anonymized healthcare records from more than 20 million people of whom more than five million are live in the system, representing $8 \%$ of the UK population [10]. Patients are representative of the UK general population in terms of age, sex and ethnicity. CPRD includes longitudinal observational data from GP electronic patient record systems in primary care practices, including medical diagnoses, referrals to specialists and to secondary care, primary care tests and investigations, lifestyle information (e.g. smoking, exercise) and prescribing data [10, 23]. Data are captured using a structured hierarchical vocabulary called Read codes [24]. Each Read code represents a health-related concept. There are $>200,000$ different codes, which are sorted into chapters (diagnoses, processes of care and medication) and subchapters [24]. Each health-related concept is represented by a 5 -byte alphanumeric code and a Read term which is the plain language description. The CPRD "Gold" dataset is drawn from the electronic patient record software Vision [25].

\section{Study population}

Patients were selected from the CPRD database according to the following specification:

1. Patients with dementia (cases) were identified by the presence of one or more dementia diagnostic codes. We adapted code lists developed by Russell et al., [26] and Rait et al., [27] (Additional file 1). The dementia code was recorded between 2000 and 2012 and the date of the first dementia code was taken as the "index date". Cases were 65 years or older at the index date and had up-to-standard records available for at least three years prior to diagnosis. All patients within the CPRD Gold dataset matching these criteria were extracted.

2. Control patients matched cases on age, sex, and general practice with three years up-to-standard data prior to the date of the matched case's index date, but had no dementia code anywhere in their patient record (up to death or end of their data collection). They were randomly sampled from the CPRD Gold dataset resulting in a 1-to-1 match between cases and controls. The index date in the controls was taken from the first diagnosis code of the matched case.

Once eligible patients had been identified, the entire available coded patient record was extracted for each patient; clinical notes and letters were not available in this dataset. This resulted in records for 95,521 individuals.

The following patients were then excluded from the dataset: cases without a matched control; cases without a dementia code within one year of their assigned index date; cases with dementia codes more than 1 year prior to the index date; controls who had a dementia code; controls prescribed medication specifically for Alzheimer's; and controls with a code for a dementia annual review. To retain the 1:1 matching, the matched case or control was also removed (See Fig. 1).

\section{Selection of model predictors}

We defined clinical entities or features a priori for this study, because of: (i) the volume of different Read codes $(60,000+$ individual codes in our dataset); (ii) the fact that there may be multiple Read codes representing the same clinical entity; and (iii) the difficulty of creating meaningful clusters of codes using data-driven methods. We drew on two sources for deciding on clinical features. First we completed a systematic review and metaanalysis of potential features from primary care records research on dementia [28]. Secondly, we carried out a consultation with 21 local GPs with the following written question: "Please could you list anything you can think of which may frequently be entered in the patient record up to 3 years before a dementia diagnosis (it does not have to be causal, just occur earlier in time than the diagnosis)." The most commonly-reported of these were: depression/low mood (suggested by 8 GPs); problem with memory (7 GPs); Fall (6 GPs); cerebrovascular accident/transient ischaemic attack (6 GPs); a 'Did not attend' code (6 GPs); high blood pressure (5 GPs); forgetful (5 GPs); and anxiety (4 GPs).

Features found to be associated with dementia in the meta-analysis were mapped together with the results of the GP survey and any features which were not readily represented by a code list were discarded (such long gaps between appointments). Also discarded were features which indicated that the process of dementia diagnosis had already been initiated by the GP (such as memory loss symptoms, cognitive screening tests, or referral to memory assessment services). Read code lists were then created to define all features. We sought code lists for these features from a clinical code list repository [29] and by emailing authors of studies included in the meta-analysis. Where code lists for features were not available, new lists were drawn up using the CPRD medical and product code 


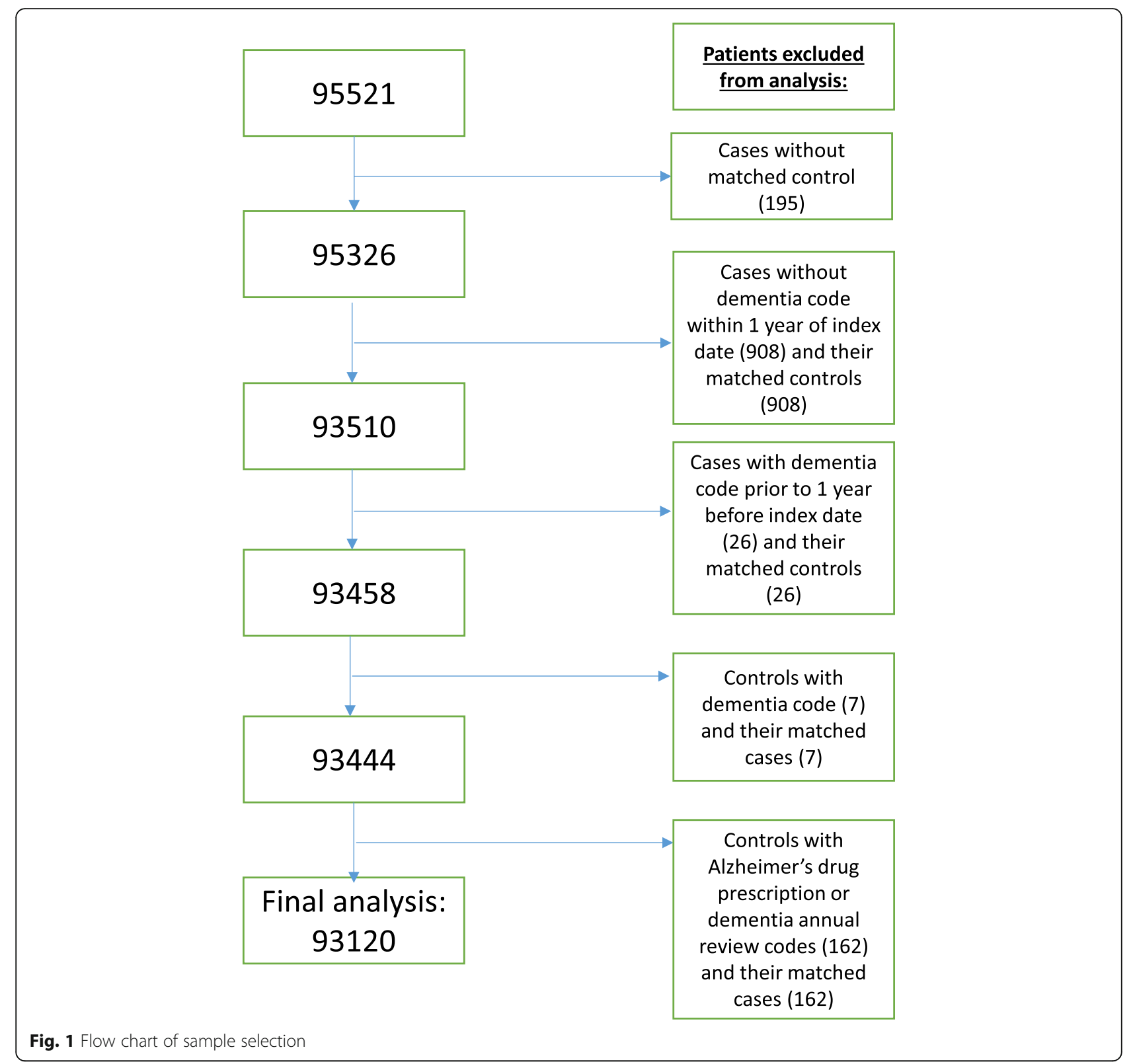

dictionaries application by authors EF and $\mathrm{RH}$ and checked by PR. This resulted in 70 code lists. (Additional file 2). Binary features were created from the code lists. The creation of binary rather than count features is thought to reduce the effect of frequency of GP visits in the data [21].

\section{Data split by time}

Code lists were matched to event-level patient data. Only data from the period five years before the index date were used. All data more than 5 years before, or at any time after, the index date were discarded. The 5-year run up period was then split into two sections representing the last year before diagnosis (year 1), to understand proximal risk factors, and the 2-5-year period before diagnosis (years 2-5), to understand static or long-standing risk factors. We ran models with each feature's data from year 1 and years 2-5 treated as a separate feature within the models (no shared variance was assumed).

\section{Data analysis}

Using a set seed to ensure the same split of patients for each model, the data were split at random into $80 \%$ for training and $20 \%$ for testing. We first ran a logistic regression model with LASSO penalisation [30]. This was our baseline statistical model, as logistic regression is the usual method for binary classification in epidemiological research, and the LASSO helped us to prioritise and 
constrain variables added to the model and allowed us to examine feature weights. We then compared further machine-learning models against this baseline method using the following algorithms:

- Random Forrest

- Naïve Bayes Classifier

- Support Vector Machines (SVM)

- Neural Networks (NN)

Data were analysed in $\mathrm{R}$ version 3.4.4 using the packages GLMnet, e1071, randomforest, pROC, ROCR, ggplot, and the neural network was run in python 2.7.12 with tensorflow 1.10.1 (Additional file 3). While tuning of various model parameters was examined, as well as more complex algorithm architectures, these offered no improvements over simpler models, therefore the most simple versions of models are presented.

Each model was assessed for its ability to classify dementia cases versus controls using the Area Under the Receiver Operating Characteristic Curve (AUROC) [31]. The values of sensitivity (recall) against specificity were examined for two values: a balanced cut-off point (sensitivity and specificity weighted equally) and a fixed specificity of 0.95 , chosen because in the clinic, it may be important to minimise false positives. Because of the case-control design of this study, we had an artificial prevalence of dementia of $50 \%$ in our sample. We thus calculated positive predictive value (precision) of each model based on the UK prevalence of dementia of $7.1 \%$ in people over 65 years [5].

The features retained within the logistic regression models following LASSO penalisation were examined, to identify the key features of the model. These were identified by generating each feature's logistic regression parameter, identified as $\beta$ in the following logistic regression equation, where $X_{n}$ indicates each feature:

$$
\operatorname{Ln}\left(\frac{P}{1-P}\right)=\beta_{0}+\beta_{1} X_{1}+\beta_{2} X_{2}+\ldots+\beta_{k} X_{k}
$$

\section{Results}

\section{Study population}

Our final sample consisted of 93,120 patients of whom 32,800 (35.2\%) were men and 60,320 (64.8\%) were women; $50 \%$ of the sample had one or more codes for dementia. The median age at index date was 82.6 years (range: 64.5-109.9 years). The median amount of time before index date available in the records was 19.3 years (range 3.00-102.2 years of registration). Dementia cases had a median of 161 events recorded in their whole record (range 2-2709) and controls had a median of 157 events recorded (range 0-2710). All patients had at least three years' worth of data (100\%), 90,351 patients
(97.0\%) had at least four years and 87,876 patients (94.4\%) at least five. 70 clinical variables were included in the model as predictors.

\section{Logistic regression and machine learning model performance}

As shown in Table 1, the logistic regression model and four further types of machine-learning models were run. Results of models can be seen in Table 1 and Fig. 2. The best AUROC was 0.74 , which was achieved by the logistic regression, the neural network and support vector machine, with the random forest model performing very similarly. The Naïve Bayes classifier model was less accurate (AUROC 0.68). The neural network gave the best specificity for a reasonable sensitivity, and thus the highest PPV.

\section{Feature weights in logistic regression model}

When the features retained by the LASSO penalisation were examined, the most important features were disorientation and wandering, behaviour change, schizophrenia, self-neglect, difficulty managing, personality change and family history of dementia; the most significant features were all recorded in the final year before diagnosis. Psychotic depression and cancer were strongly negatively associated with dementia (Table 2).

\section{Discussion}

\section{Summary of findings}

Our study gives new insights into the possibilities of identifying undetected cases of dementia in primary care by using GP patient records as the sole data source. We found that LASSO penalised logistic regression, support vector machine, neural network and random forest models performed very similarly, with a best AUROC of 0.74, although the neural network produced the highest PPV (precision; 0.31). Logistic regression and random forest algorithms may nevertheless offer an advantage over support vector machines and neural networks as they produce easy to interpret feature weights, which may be of value in a clinical situation.

\section{Important features in the model}

In this study, the important features found by the logistic regression were intuitively important clinically and were either symptoms which indicated the patient was already in the prodromal stages of dementia or indications of increasing frailty. Symptoms such as disorientation and wandering, behaviour or personality change; medications such as antidepressants and antipsychotics; observations such as self-neglect and difficulty managing; and administrative codes such as 'third party consultation', 'did not attend' and 'GP home visit', were all among the top 15 features. In this regard our study is novel, compared to other primary care dementia risk 
Table 1 Model performance (AUROC, best sensitivity and specificity, PPV)

\begin{tabular}{|c|c|c|c|c|c|c|c|}
\hline Model Type & $\begin{array}{l}\text { Time } \\
\text { split }\end{array}$ & $\begin{array}{l}\text { AUROC } \\
(95 \% \mathrm{Cl})\end{array}$ & $\begin{array}{l}\text { Specificity (balanced } \\
\text { model) }\end{array}$ & $\begin{array}{l}\text { Sensitivity (balanced } \\
\text { model) }\end{array}$ & $\begin{array}{l}\text { PPV (balanced } \\
\text { model) }\end{array}$ & $\begin{array}{l}\text { Sensitivity for } \\
95 \% \text { specificity }\end{array}$ & $\begin{array}{l}\text { PPV at } 95 \% \\
\text { specificity }\end{array}$ \\
\hline Logistic Regression with Lasso & $1,2-5$ & $0.736(0.728-0.743)$ & 0.752 & 0.602 & 0.156 & 0.222 & 0.254 \\
\hline Naïve Bayes Classifier & $1,2-5$ & $0.682(0.675-0.690)$ & 0.906 & 0.241 & 0.164 & 0.153 & 0.189 \\
\hline Support Vector Machine & $1,2-5$ & $0.737(0.730-0.744)$ & 0.691 & 0.674 & 0.142 & 0.223 & 0.255 \\
\hline Random Forest & $1,2-5$ & $0.734(0.726-0.740)$ & 0.653 & 0.700 & 0.134 & 0.210 & 0.239 \\
\hline Neural Network ( $3 \times 139$ nodes) & $1,2-5$ & $0.737(0.730-0.743)$ & 0.781 & 0.619 & 0.178 & 0.298 & 0.312 \\
\hline
\end{tabular}

prediction models, due to its expanded list of symptom and administrative features. Our aim was to identify undetected, but current cases of dementia, rather than predict onset at some future time. We thus took an approach of using clinical entities that may be associated with dementia for any reason, appearing in the patient record prior to or around the time of dementia onset, rather than restricting ourselves to entities with a causal relationship to dementia. Future work could examine at which time point, prior to dementia diagnosis, each of these features starts contributing significantly to the model.

\section{Performance of machine-learning over traditional methods} Our study offers an improvement on previous models which aim to predict or detect dementia using GP patient record data as the only source of information, by using an expanded list of predictors and achieving a best
PPV of 0.31. Walters et al. [19], retained 14 clinical and demographic variables using Cox proportional hazards regression with backwards elimination in a cohort design. Their model showed similar sensitivity to ours but higher specificity, and a best PPV of only 0.11 . Their model included age as one of the features, which is likely to be one of the best predictors of dementia, and which our matched case-control design did not allow for. Our model may thus show better performance if replicated in a cohort sample, adding in age as a predictor.

We found that machine-learning models showed no improvement over a logistic regression method which was allowed to select features using a data-driven mechanism. Some machine learning techniques allow for non-linear effects to be learned in the data, whereas logistic regression assumes linear relationships between variables. This freedom of the models to find non-linear

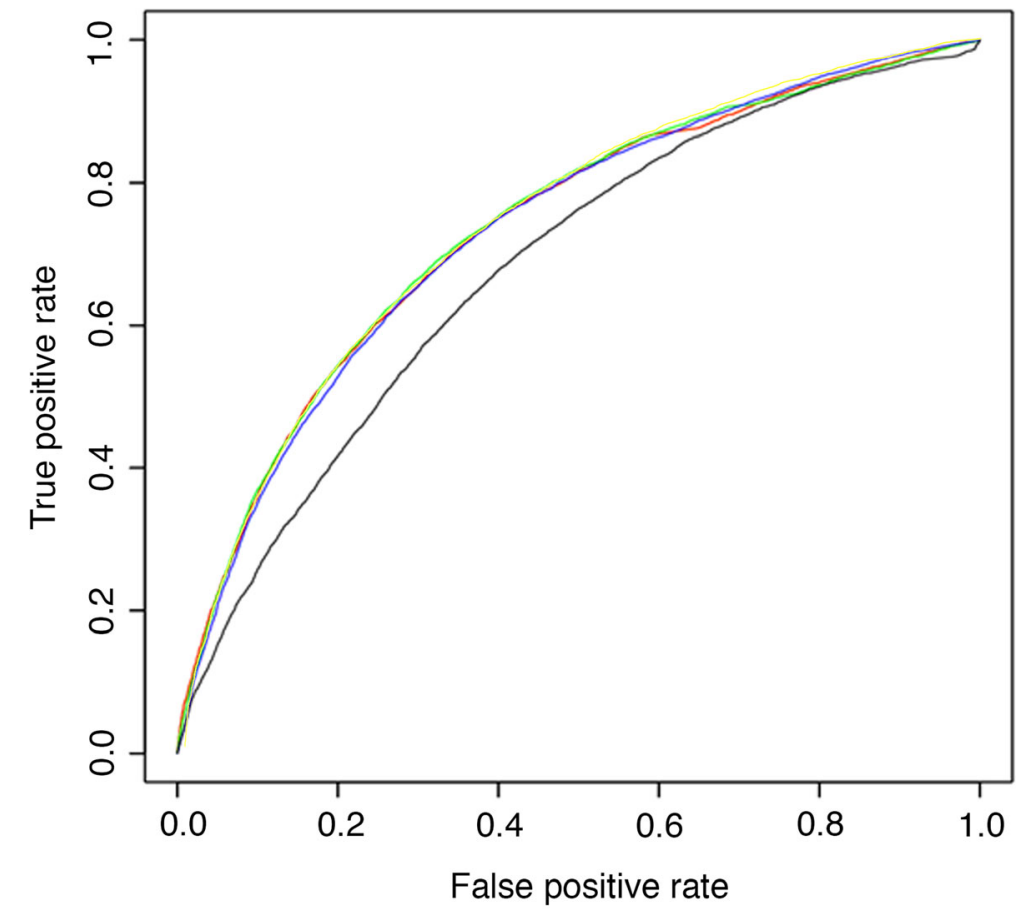

Key: Red = logistic regression; Green = SVM; Blue = Random Forest;

Black $=$ Naïve Bayes; Yellow $=$ Neural Network

Fig. 2 AUROC for all ML models superimposed; 1, 2-5 year data 
Table 2 Features retained in Logistic Regression with Lasso Penalisation, 1 year and $2-5$ years separated

\begin{tabular}{|c|c|c|}
\hline \multirow{2}{*}{$\begin{array}{l}\text { Feature name } \\
1 \text { year prior to diagnosis/ } \\
\text { matched date }\end{array}$} & \multicolumn{2}{|c|}{ Logistic regression parameter } \\
\hline & $\begin{array}{l}1 \text { year prior to diagnosis/ } \\
\text { matched date }\end{array}$ & $\begin{array}{l}2-5 \text { year } \\
\text { predictors }\end{array}$ \\
\hline Disorientation and Wandering & 2.31 & 0.88 \\
\hline Behaviour change & 1.99 & 0.65 \\
\hline Schizophrenia & 1.53 & - \\
\hline Self-neglect & 1.45 & - \\
\hline Difficulty managing & 1.38 & - \\
\hline Personality change & 1.18 & 0.58 \\
\hline Family history of dementia & 1.14 & - \\
\hline Third party consultation & 0.85 & - \\
\hline Antidepressant & 0.81 & - \\
\hline Antipsychotic medication & 0.76 & -0.11 \\
\hline Cerebrovascular disease & 0.58 & 0.14 \\
\hline Did not attend & 0.56 & 0.22 \\
\hline GP home visit & 0.55 & -0.11 \\
\hline Bipolar disorder & 0.51 & -0.11 \\
\hline Interaction with social services & 0.51 & - \\
\hline Possible Fall & 0.47 & 0.22 \\
\hline Alcohol & 0.42 & - \\
\hline Unable to cope & 0.41 & 0.21 \\
\hline Attended Emergency Department & 0.39 & - \\
\hline Depression & 0.34 & - \\
\hline Living in a nursing home & 0.31 & - \\
\hline Receiving care in home & 0.28 & - \\
\hline Epilepsy or Seizures & 0.23 & 0.25 \\
\hline Blood pressure measurement & 0.16 & - \\
\hline Stroke & 0.15 & - \\
\hline Routine hospital admission & 0.15 & -0.14 \\
\hline Z-drugs & 0.13 & -0.11 \\
\hline Lower limb fracture & 0.12 & - \\
\hline Receiving care in home & 0.11 & - \\
\hline Anxiety & 0.10 & - \\
\hline Impaired mobility & 0.10 & - \\
\hline $\begin{array}{l}\text { Needs help with activities of daily } \\
\text { living }\end{array}$ & -0.11 & -0.30 \\
\hline Dressing of wound, burn or ulcer & -0.13 & - \\
\hline Family bereavement & -0.16 & - \\
\hline Hypertension & -0.20 & -0.16 \\
\hline Infections & -0.21 & -0.16 \\
\hline Angina & -0.22 & - \\
\hline Vertebral collapse & -0.27 & - \\
\hline Lithium & -0.28 & - \\
\hline PTSD reaction & -0.46 & - \\
\hline Cancer & -1.06 & - \\
\hline
\end{tabular}

Table 2 Features retained in Logistic Regression with Lasso Penalisation, 1 year and 2-5 years separated (Continued)

\begin{tabular}{|c|c|c|}
\hline \multirow{2}{*}{$\begin{array}{l}\text { Feature name } \\
1 \text { year prior to diagnosis/ } \\
\text { matched date }\end{array}$} & \multicolumn{2}{|c|}{ Logistic regression parameter } \\
\hline & $\begin{array}{l}1 \text { year prior to diagnosis/ } \\
\text { matched date }\end{array}$ & $\begin{array}{l}2-5 \text { year } \\
\text { predictors }\end{array}$ \\
\hline Psychotic Depression & -1.11 & - \\
\hline Personality disorder & - & 0.21 \\
\hline Constipation & - & 0.10 \\
\hline Coronary Heart Disease & - & -0.12 \\
\hline Obesity & - & -0.16 \\
\hline Benzodiazepines & - & -0.22 \\
\hline
\end{tabular}

effects did not seem to improve the discriminatory power. It may be that electronic health records data are too noisy to achieve much improvement by using newer methods over traditional methods, or it may be that all the relationships between variables in the model are best approximated with linear relationships.

With no better performance of one model over another, it is worth considering that clinicians appreciate knowing the reasons behind decisions reached by computer-aided decision support algorithms [32], to allow them autonomy and flexibility in the use of such algorithms [33]. Indeed, the new legislation on use of personal data (GDPR) may require that decisions based on processing of personal or patient data allow for transparent interpretation of how results are reached [34]. Thus the "black box" of the neural network could prove a barrier to clinical implementation [35]. While there are ways of recovering the choices or reasoning behind neural networks, these are not yet robust or reliable, especially in EHR data. Logistic regressions and random forest algorithms allow for important features to be exposed, thus aiding clinical interpretation of the algorithm classification decision, and may be the best approaches for prediction tasks which aim to be implemented in the clinic.

\section{Clinical implications}

Our findings are a useful development of the evidence base for generating a system that can be applied to identify undiagnosed cases of dementia from primary care electronic records. Our broad approach and the elements in our model can be used and contribute to further research to create a detection tool for GPs, commissioners, or public health service planners. Our findings, taken with that generated by other groups using similar methodologies, make clear that an algorithmic approach alone is not able to make the diagnosis of dementia or identify those with dementia by itself. Future approaches are likely to use systems such as this to flag up cases where GPs can offer further clinical evaluation at the patient's next primary care consultation. The role of primary care at this point might be to identify cases that would benefit from definitive 
assessment in a Memory Assessment Service, taking account of patient preference for such an assessment. After further development of our model, the next step could be pilot testing of an implemented decision support tool that is triggered to ask a GP to consider a review, request a diagnosis code, or ask the GP to ask a patient about falls and other safety issues, or even fill in more detail in the record to improve the risk estimate. A further potential use would be for service planners who wish to estimate local area prevalence of dementia, so that Memory Assessment Services can be commissioned appropriately. The next steps in developing this model should include consultation with general practitioners, patients, and commissioners, to understand stakeholder priorities for improving the model for implementation and early detection. One priority for such stakeholders might be to have the model produce a personalised risk estimate for dementia. This would mean that the response by the GP could be tailored to the patient's individual circumstances, rather than a one-size-fits-all approach.

\section{Strengths and limitations}

The key strength to our approach was the comprehensive strategy for identifying a wide range of potential features for the model, from clinically related diagnoses, health events and symptoms, to more social or administrative features, which may be recorded as the GP takes care of the wider needs of the patient.

Limitations include the case-control design. A cohort design would have been closer to a real life or clinic setting in terms of prevalence of dementia; in addition, age could have been included as a predictor. The model presented here should be replicated in a cohort dataset to examine its fit to a novel data set, and to refine it further. A second limitation is that static binary features may have resulted in a loss of information, although where we have previously trialled "count" features, these have not improved the accuracy of our models. Other studies have also favoured binary features in order to reduce the influence on the data of the number of times a patient visits their GP [21]. A third limitation is that we did not inform the model that variables representing the same feature measured at different times were related to each other. This could be achieved by using a multi-level model which treats yearly features as a cluster of predictors which share variance.

\section{Future directions}

Using a comprehensive list of features we achieved a fair discrimination between cases and controls which could aid with local prevalence estimates and, particularly, estimates that are based on detailed local information, and early detection. Given our methodical approach to selecting predictive features, we believe that this model provides a strong basis for further development with more sophisticated feature engineering. Our team's future work will explore longitudinal information within patient records to identify how much earlier the diagnosis could be made, and how the best set of features evolve in the time period before diagnosis. We will also explore the inclusion of features which indicate a change in memory or cognitive function over time, such as missed appointments becoming more common.

Many cases of dementia which are apparently undiagnosed are actually detected by GPs but unlabelled due to a lack of a formal diagnosis. These 'detected but unlabelled' patients may make up a substantial proportion of undiagnosed patients with dementia, as many GPs are not convinced of the benefit of a formal dementia diagnosis [36, 37]. Creating a model to find these unlabelled cases may allow for more sensitive detection of participants for clinical trials, as well as improving the quality of GP record keeping for audit, health service planning and prevalence studies.

\section{Conclusions}

We successfully discriminated between dementia cases and controls using only features from the primary care record which did not indicate that memory problems had already been detected by GPs. We found no advantage of newer machine learning techniques over logistic regression. We identified the most important features for detecting dementia in such a model, these were found to be possible prodromal symptoms and indications of increasing frailty. With further development and as part of a comprehensive diagnostic pathway, this model may aid GPs and health service planners with the early detection of dementia in primary care.

\section{Supplementary information}

Supplementary information accompanies this paper at https://doi.org/10. 1186/s12911-019-0991-9.

Additional file 1. Dementia Read Codes.

Additional file 2. Feature List.

Additional file 3. Model Specifications.

\section{Abbreviations \\ AUROC: Area Under the Receiver Operating Characteristic Curve; Cl: Confidence Interval; CPRD: Clinical Practice Research Datalink; EPR: Electronic Patient Record; GDPR: General Data Protection Regulation; GP: General Practitioner/General Practice; LASSO: Least Absolute Shrinkage and Selection Operator; MMSE: Mini-Mental State Examination; NHS: National Health Service; NN: Neural Network; PPV: Positive Predictive Value; SVM: Support Vector Machine; UK: United Kingdom of Great Britain and Northern Ireland}

\section{Acknowledgements}

This work uses data provided by patients and collected by the NHS as part of their care and support. \#datasaveslives.

\section{Authors' contributions}

EF, SO and JC conceived and directed the study. EF and RH drew up code lists and PR checked these. PR managed the data and conducted the 
analyses. PH and SO gave data analysis advice. SB, JC, HvM and RH gave clinical advice. EF wrote the manuscript. All authors provided critical feedback on the manuscript and approved the final version.

\section{Funding}

This project was funded by a grant from the Wellcome Trust ref. 202133/Z/ $16 / Z$. The funder had no role in study design, data collection and analysis, decision to publish, or preparation of the manuscript.

\section{Availability of data and materials}

The data that support the findings of this study are available from Clinical Practice Research Datalink (CPRD; www.cprd.com) but restrictions apply to the availability of these data, which were used under license for the current study, and so are not publicly available. For re-using these data, an application must be made directly to CPRD.

\section{Ethics approval}

This study was approved by the Independent Scientific Advisory Committee at the Medicines and Healthcare Products Regulatory Authority, UK, protocol number 15_111_R. Following approval, administrative permissions to access and use the electronic patient records were granted by Clinical Practice Research Datalink (CPRD.com).

Consultations with stakeholders (GPs) for guiding the design of research do not need ethics approvals or written consent.

\section{Consent for publication}

For EHR data: Not applicable

\section{Competing interests}

The authors declare that they have no competing interests.

\section{Author details}

${ }^{1}$ Department of Primary Care and Public Health, Brighton and Sussex Medical School, Watson Building, Village Way, Falmer, Brighton BN1 9PH, England. ${ }^{2}$ Department of Physics and Astronomy, University of Sussex, Brighton BN1 $9 R Q$, England. ${ }^{3}$ Faculty of Health, University of Plymouth, Plymouth PL4 8AA, England.

Received: 5 June 2019 Accepted: 21 November 2019

Published online: 02 December 2019

\section{References}

1. Banerjee S. The use of antipsychotic medication for people with dementia: time for action. London: Department of Health; 2009

2. Living well with dementia: A National Dementia Strategy [https:/www.gov.uk/ government/uploads/system/uploads/attachment_data/file/168220/dh_094051.pdf].

3. Banerjee $\mathrm{S}$. The macroeconomics of dementia-will the world economy get Alzheimer's disease? Arch Med Res. 2012;43(8):705-9.

4. Dementia [https://www.england.nhs.uk/mental-health/dementia/].

5. Prince $M$, Knapp M, Guerchet $M$, McCrone P, Prina M, Comas-Herrera A, Wittenberg R, Adelaja B, Hu B, King D et al: Dementia UK Update In. Edited by Society As, vol. Second Edition. London, UK; 2014

6. Pentzek M, Wollny A, Wiese B, Jessen F, Haller F, Maier W, Riedel-Heller SG, Angermeyer MC, Bickel $\mathrm{H}$, Mosch $\mathrm{E}$, et al. Apart from nihilism and stigma: what influences general practitioners' accuracy in identifying incident dementia? The American journal of geriatric psychiatry : official journal of the American Association for Geriatric Psychiatry. 2009;17(11):965-75.

7. Connolly A, Gaehl E, Martin H, Morris J, Purandare N. Underdiagnosis of dementia in primary care: variations in the observed prevalence and comparisons to the expected prevalence. Aging Ment Health. 2011;15(8):978-84.

8. Dementia diagnosis rate workbooks [https://www.england.nhs.uk/ publication/dementia-diagnosis-rate-workbook/].

9. Prince M, Bryce R, Ferri C: World Alzheimer report 2011: the benefits of early diagnosis and intervention: Alzheimer's disease international; 2011.

10. Herrett E, Gallagher AM, Bhaskaran K, Forbes H, Mathur R, van Staa T, Smeeth L. Data resource profile: clinical practice research Datalink (CPRD). Int J Epidemiol. 2015;44(3):827-36.

11. Ghosh RE, Crellin E, Beatty S, Donegan K, Myles P, Williams R. How clinical practice research Datalink data are used to support pharmacovigilance. Ther Adv Drug Saf. 2019;10:2042098619854010.
12. Bamford C, Eccles M, Steen N, Robinson L. Can primary care record review facilitate earlier diagnosis of dementia? Fam Pract. 2007:24:108-16.

13. Ramakers IH, Visser PJ, Aalten P, Boesten JH, Metsemakers JF, Jolles J, Verhey FR. Symptoms of preclinical dementia in general practice up to five years before dementia diagnosis. Dement Geriatr Cogn Disord. 2007;24(4):300-6.

14. Stephan BC, Kurth T, Matthews FE, Brayne C, Dufouil C. Dementia risk prediction in the population: are screening models accurate? Nat Rev Neurol. 2010;6(6):318.

15. Stephan B, Brayne C. Risk factors and screening methods for detecting dementia: a narrative review. J Alzheimers Dis. 2014;42(s4):S329-38.

16. Stephan BC, Tang E, Muniz-Terrera G. Composite risk scores for predicting dementia. Curr Opin Psychiatry. 2016;29(2):174-80.

17. Tang EYH, Harrison SL, Errington L, Gordon MF, Visser PJ, Novak G. Current developments in dementia risk prediction modelling: an updated systematic review. PLoS One. 2015:10(9):e0136181.

18. The PHG Foundation: Dementia Risk Prediction Models: What do policy makers need to know? In. Cambridge, UK: The University of Cambridge; 2019.

19. Walters K, Hardoon S, Petersen I, lliffe S, Omar RZ, Nazareth I, Rait G. Predicting dementia risk in primary care: development and validation of the dementia risk score using routinely collected data. BMC Med. 2016;14(1):1-12.

20. Jessen F, Wiese B, Bickel H, Eifflander-Gorfer S, Fuchs A, Kaduszkiewicz H. Prediction of dementia in primary care patients. PLoS One. 2011:6.

21. Jammeh EA, Camille BC, Stephen WP, Escudero J, Anastasiou A, Zhao P, Chenore T, Zajicek J, Ifeachor E. Machine-learning based identification of undiagnosed dementia in primary care: a feasibility study. BJGP open. 2018; 2(2):bjgpopen18X101589.

22. Clinical Practice Research Datalink [www.cprd.com].

23. Williams T, Van Staa T, Puri S, Eaton S. Recent advances in the utility and use of the general practice research database as an example of a UK primary care data resource. Ther Adv Drug Saf. 2012;3(2):89-99.

24. Chisholm J. The read clinical classification. BMJ. 1990;300:1092.

25. [https://www.visionhealth.co.uk/vision-medical-software/]

26. Russell P, Banerjee S, Watt J, Adleman R, Agoe B, Burnie N, Carefull A, Chandan $\mathrm{K}$, Constable D, Daniels $\mathrm{M}$, et al. Improving the identification of people with dementia in primary care: evaluation of the impact of primary care dementia coding guidance on identified prevalence. BMJ Open. 2013;3(12):e004023.

27. Rait G, Walters K, Bottomley C, Petersen I, Niffe S, Nazareth I. Survival of people with clinical diagnosis of dementia in primary care: cohort study. $\mathrm{Br}$ Med J. 2010;341:c3584.

28. Ford E, Greenslade N, Paudyal P, Bremner S, Smith HE, Banerjee S, Sadhwani S, Rooney P, Oliver S, Cassell J. Predicting dementia from primary care records: a systematic review and meta-analysis. PLoS One. 2018;13(3):e0194735.

29. [https://clinicalcodes.rss.mhs.man.ac.uk/]

30. Tibshirani R. Regression shrinkage and selection via the lasso. J R Stat Soc Ser B Methodol. 1996;58(1):267-88.

31. Mandrekar JN. Receiver operating characteristic curve in diagnostic test assessment. J Thorac Oncol. 2010;5(9):1315-6.

32. Fiks AG. Designing computerized decision support that works for clinicians and families. Curr Probl Pediatr Adolesc Health Care. 2011;41(3):60-88.

33. Trivedi MH, Daly EJ, Kern JK, Grannemann BD, Sunderajan P, Claassen CA. Barriers to implementation of a computerized decision support system for depression: an observational report on lessons learned in "real world" clinical settings. BMC Medical Inform Decis Making. 2009;9(1):6.

34. Moerel L, Storm M: Automated decisions based on profiling: information, explanation or justification-that is the question! Autonomous Systems and the Law (2019) Editors: Nikita Aggarwal, Horst Eidenmüller, Luca Enriques, Jennifer Payne, Kristin van Zwieten Beck CH 2019.

35. GPRD and research - An overview for researchers [https://www.ukri.org/aboutus/policies-and-standards/gdpr-and-research-an-overview-for-researchers/]

36. Cahill S, Clark M, O'connell H, Lawlor B, Coen R, Walsh C. The attitudes and practices of general practitioners regarding dementia diagnosis in Ireland. International Journal of Geriatric Psychiatry. J Psychiatry Late Life Allied Sci. 2008:23(7):663-9.

37. van Hout H, Vernooij-Dassen M, Bakker K, Blom M, Grol R. General practitioners on dementia: tasks, practices and obstacles. Patient Educ Couns. 2000:39(2-3):219-25.

\section{Publisher's Note}

Springer Nature remains neutral with regard to jurisdictional claims in published maps and institutional affiliations. 